\title{
Spectroscopic confirmation of the low-latitude object FSR 1716 as an old globular cluster
}

\author{
Andreas Koch ${ }^{1,2}$, Andrea Kunder ${ }^{3,4}$, and Jennifer Wojno ${ }^{4}$ \\ 1 Zentrum für Astronomie der Universität Heidelberg, Astronomisches Recheninstitut, Mönchhofstr. 12, 69120 Heidelberg, Germany \\ e-mail: a. koch1@lancaster.ac.uk \\ 2 Department of Physics, Lancaster University, LA1 4YB, Lancaster, UK \\ 3 Saint Martin's University, Old Main, 5000 Abbey Way SE, Lacey, WA 98503, USA \\ ${ }^{4}$ Leibniz-Institut für Astrophysik Potsdam, An der Sternwarte 16, 14482 Potsdam, Germany
}

Received 14 August 2017 / Accepted 26 August 2017

\begin{abstract}
Star clusters are invaluable tracers of the Galactic components and the discovery and characterization of low-mass stellar systems can be used to appraise their prevailing disruption mechanisms and time scales. However, owing to significant foreground contamination, high extinction, and still uncharted interfaces of the underlying Milky Way components, objects at low Galactic latitudes are notoriously difficult to characterize. Here, we present the first spectroscopic campaign to identify the chemodynamical properties of the low-latitude star cluster FSR 1716. While its photometric age and distance are far from settled, the presence of RR Lyrae variables indicates a rather old cluster variety. Using medium-resolution $(R \sim 10600)$ calcium triplet $(\mathrm{CaT})$ spectroscopy obtained with the wide-field, multi-fiber AAOmega instrument, we identified six member candidates with a mean velocity of $-30 \mathrm{~km} \mathrm{~s}^{-1}$ and a velocity dispersion of $2.5 \pm 0.9 \mathrm{~km} \mathrm{~s}^{-1}$. The latter value implies a dynamic mass of $\sim 1.3 \times 10^{4} M_{\odot}$, typical of a low-mass globular cluster. Combined with our derived CaT metallicity of $-1.38 \pm 0.20 \mathrm{dex}$, this object is finally confirmed as an old, metal-poor globular cluster.
\end{abstract}

Key words. stars: abundances - Galaxy: abundances - Galaxy: structure - Galaxy: disk - open clusters and associations: general globular clusters: individual: FSR 1716

\section{Introduction}

Star clusters in any of the Galactic components (halo, disks, bulge) are crucial tracers of the prevailing stellar populations at their birthplace. Thus they play an important role for disentangling the chemodynamic evolution of the Milky Way. For a unique mapping it is therefore imperative to be able to distinguish between low-mass, young objects (e.g., Vande Putte et al. 2010) and old globular clusters (GCs), or to diversify objects formed in situ versus those accreted from larger objects such as dwarf galaxies (Marín-Franch et al. 2009; Law \& Majewski 2010). This becomes progressively more difficult towards the interfaces of the components, when distinctions between, for example, the inner and outer halo or the disk-bulge-halo transition need to be sampled (e.g., Recio-Blanco et al. 2014; Koch et al. 2016, 2017a; Mucciarelli et al. 2017).

However, such a cartography becomes challenging towards the plane of the Milky Way, since the high extinction and confusion with disk field stars along the line of sight render such studies a "pain in the plane". Past estimates of the completeness of GCs in the Milky Way (e.g., Ivanov et al. 2005) indicated that about ten $( \pm 3)$ clusters remain missing towards the inner Milky Way, and that the discovery of more unknown objects still lurking in the Galactic anticenter direction would add invaluable information to the overall population of Galactic star clusters. Once discovered, it needs to be carefully assessed which Galactic component has spawned them, but without the proper chemical and/or kinematic information, this endeavour remains aleatoric.
Here, we continue our efforts to characterize hitherto uncharted star clusters in the Milky Way (Koch et al. 2017a,b) by performing a spectroscopic study of the low-latitude $\left(l=330^{\circ}\right.$, $b=-1.6^{\circ}$ ) object FSR 1716. This star cluster was discovered by Froebrich et al. (2007) in the infrared Two Micron All Sky Survey (2MASS; Cutri et al. 2003). Subsequent analysis of ESO NTT infrared (JHK) data indicated an age of $>2$ Gyr and a metalpoor nature of approximately -1.6 dex (Froebrich et al. 2008). Since then, no consensus had been reached on whether this is an open or a globular cluster.

Upon re-analysis of the 2MASS data, Bonatto \& Bica (2008) confirmed an old age between 7 and $12 \mathrm{Gyr}$ and placed FSR 1716 within $0.8-2.3 \mathrm{kpc}$ of the Solar radius ${ }^{1}$. However, in order to remain stable at that old age, this system needed to be an "extreme dynamic survivor", since the severe disruption mechanisms in the Galactic disk would lead to strong mass loss. Accordingly, open clusters as old as this are mainly found at larger Galactocentric distances due to the decreased relentlessness of dynamical processes leading to their fast disruptions on time scales well below 1 Gyr (Lamers et al. 2005; Bonatto \& Bica 2007; Friel 2013). Their derived very small radius $\left(r_{t}<4 \mathrm{pc}\right)$, low stellar content $\left(M<200 M_{\odot}\right)$, and Solar metallicity prompted Bonatto \& Bica (2008) to prefer characterization as a rare, old open cluster over a low-mass, old globular cluster. In contrast, Buckner \& Froebrich (2016) assigned this

\footnotetext{
1 We note that this estimate used a value for the Solar radius of $7.2 \mathrm{kpc}$, whereas new measurements place this at larger distances of $\sim 8.3 \mathrm{kpc}$ (e.g., Reid et al. 2009; Brunthaler et al. 2011).
} 
Table 1. Photometric properties of the FSR 1716 member candidates.

\begin{tabular}{cccccccc}
\hline \hline Star & $\begin{array}{c}\text { ID } \\
(2 M A S S)\end{array}$ & $\begin{array}{c}\alpha \\
(\mathrm{J} 2000.0)\end{array}$ & $\begin{array}{c}\delta \\
(\mathrm{J} 2000.0)\end{array}$ & $\begin{array}{c}r \\
{\left[{ }^{\prime \prime}\right]}\end{array}$ & $\begin{array}{c}J \\
{[\mathrm{mag}]}\end{array}$ & $\begin{array}{c}H \\
{[\mathrm{mag}]}\end{array}$ & $\begin{array}{c}K \\
{[\mathrm{mag}]}\end{array}$ \\
\hline Plate 1 \#16 & $\mathrm{J} 161030.35-534450.8$ & $16: 10: 30.35$ & $-53: 44: 50.81$ & 6.0 & 13.429 & 12.393 & 12.013 \\
Plate 1 \#24 & $\mathrm{J} 161032.22-534550.3$ & $16: 10: 32.22$ & $-53: 45: 50.33$ & 57.8 & 12.710 & 11.547 & 11.189 \\
Plate 1 \#106 & $\mathrm{J} 161025.78-534446.3$ & $16: 10: 25.78$ & $-53: 44: 46.28$ & 38.7 & 13.971 & 12.954 & 12.641 \\
Plate 2 \#266 & $\mathrm{J} 161026.77-534407.7$ & $16: 10: 26.77$ & $-53: 44: 07.73$ & 56.1 & 13.858 & 12.840 & 12.395 \\
Plate 3 \#76 & $\mathrm{J} 161029.88-534605.4$ & $16: 10: 29.88$ & $-53: 46: 05.35$ & 69.4 & 12.897 & 11.737 & 11.333 \\
Plate 3 \#213 & $\mathrm{J} 161026.46-534423.2$ & $16: 10: 26.46$ & $-53: 44: 23.18$ & 45.4 & 14.435 & 13.426 & 13.069 \\
\hline
\end{tabular}

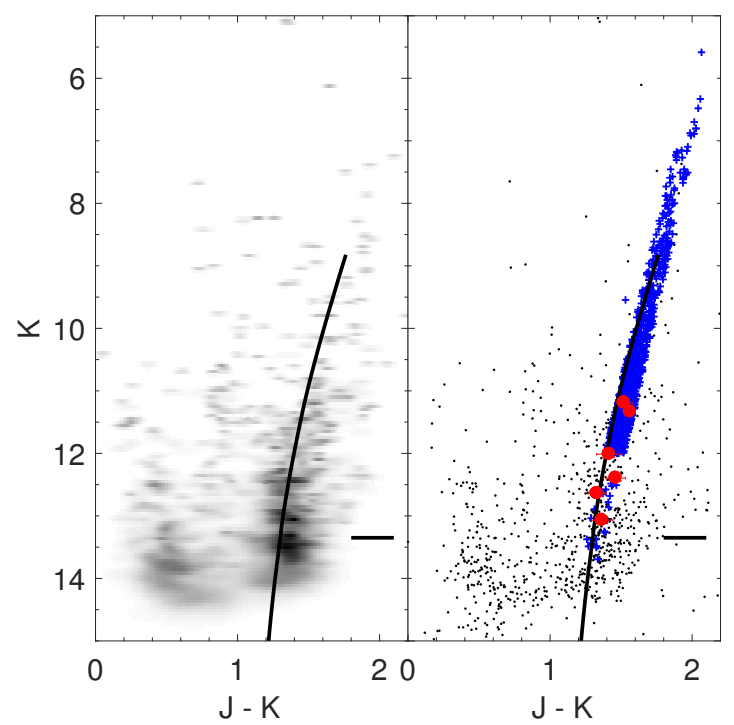

Fig. 1. Left panel: Hess diagram of stars within $4^{\prime}$ of the nominal cluster center using 2MASS photometry. Right panel: member candidates as red points, whereas blue crosses indicate foreground stars that were used as fiber-filler targets. In either case, we highlight the red clump level at $K_{\mathrm{S}}=13.35 \mathrm{mag}$ and a Dartmouth isochrone (Dotter et al. $2008)$, using the best parameters for a reddening $\left(A_{K}=0.38 \mathrm{mag}\right)$, distance (14.62 mag), age (10 Gyr), and metallicity ( $-1.5 \mathrm{dex})$ from Minniti et al. (2017).

object an old age (10-12 Gyr), but at a larger distance to the Sun of $7.3 \mathrm{kpc}$.

Recently, FSR 1716 was identified via its RR Lyrae population in the deep, multi-epoch, near-infrared "VISTA Variables in the Vía Láctea" (VVV) survey (Minniti et al. 2017), which ascertained an old age of $>10$ Gyr and a low metallicity $(-1.5$ dex $)$ from the RR-Lyrae's period-metallicity relation. Accordingly, this analysis placed FSR 1716 at a distance well within the Solar circle, at $d_{\odot}=7.5 \mathrm{kpc}$ and $R_{\mathrm{GC}}=4.3 \mathrm{kpc}$. Taken at face value, these values would argue in favor of this object indeed being of the globular variety. Therefore, only the addition of chemical abundance and detailed kinematic information will be able to settle the true nature of FSR 1716 and its proper place in the Milky Way galaxy.

This paper is organized as follows: in Sect. 2 we describe our spectroscopic observations, from which we measure the stellar kinematics and assess membership with the star cluster in Sect. 3. Section 4 contains our derivation of metallicities, and finally, in Sect. 5, we summarize our findings.

\section{Observations}

Targets for this program were selected from the 2MASS cata$\log$, applying generous selection boxes that would, in principle, encompass the cluster's red giant branch (RGB) from its tip $\left(K_{\mathrm{S}} \sim 9 \mathrm{mag}\right.$ ) to its red clump level (at $K_{\mathrm{S}} \sim 13.5 \mathrm{mag}$ ). Owing to its very low latitude, the line of sight towards FSR 1716 is heavily contaminated with Galactic disk stars, which renders an efficient target selection difficult (Fig. 1; see also Fig. 4 in Minniti et al. 2017). Similarly, the success rate of our procedure is limited by the small extent of the cluster: at the distance to FSR 1716 of $\sim 7.5 \mathrm{kpc}$, its core radius subtends a mere $26.5^{\prime \prime}$ (Froebrich et al. 2008), while Minniti et al. (2017) estimate a full extent of the cluster of $\sim 3^{\prime}$. This compares to a field of view of the AAOmega instrument of $2^{\circ}$ and a minimum fiber separation of $30^{\prime \prime}$ (Sharp et al. 2006). Details on the bona fide member candidates, as determined in Sect. 3, are summarized in Table 1.

The data presented here were taken over three nights during our six-nights' observing run in June 2017, with the AAOmega multifibre spectrograph at the $3.9 \mathrm{~m}$ Anglo-Australian Telescope (Siding Spring Observatory, Coonabarabran, NSW, Australia). We created three plate configurations for the Two Degree Field (2dF) fibre positioner, containing a total of 1048 science fibres and 75 sky positions. As before, we used a dual setup that employed the red $1700 \mathrm{D}$ grating, centered at $8600 \AA$ and the blue $2500 \mathrm{~V}$ grating, centered at $5000 \AA$. This way we were sure to comprise the prominent calcium triplet (CaT) lines in the red. In the following, we only employ the red part of the spectra owing to the overly low signal in our targets at the bluest wavelengths. The exposure times were $3 \times 1200 \mathrm{~s}$ for plates 1 and 3 and $2 \times 1500 \mathrm{~s}$ for plate 2 , with overall seeing conditions of $\sim 1.1-1.4^{\prime \prime}$. Data reduction was carried out in a standard manner using AAO's 2dfdr pipeline (AAO software Team 2015), which performs quartz-flatfielding, wavelength calibration via arc-lamp exposures, sky subtraction using the dedicated sky fibers, and optimal extraction of the science spectra. The final wavelength range is $8350-8800 \AA$, with slight variations depending on the exact position of the spectra on the CCD. Our spectra reach a resolving power of $R \sim 10600$, as measured from the width of the calibration arc lamps. Finally, the median signal-to-noise ratio $(\mathrm{S} / \mathrm{N})$ of our final extracted spectra is $30 \mathrm{px}^{-1}$ on the red chip, with individual values reaching $\sim 10-70 \mathrm{px}^{-1}$. While, in similar programs of higher $\mathrm{S} / \mathrm{N}$, we were able to determine chemical abundance ratios of several $\alpha$ - and heavy elements in GC stars (Koch et al. 2017a), this was not achievable from the present data set.

\section{Radial velocities, cluster membership, and dynamical mass}

Radial velocities were measured via cross-correlation of the three strong CaT lines against a synthetic template of that region (Kleyna et al. 2004), which led to a median velocity error of $1.8 \mathrm{~km} \mathrm{~s}^{-1}$. In Fig. 2 we show the run of velocities versus radial distance from the nominal cluster center, here parameterized 


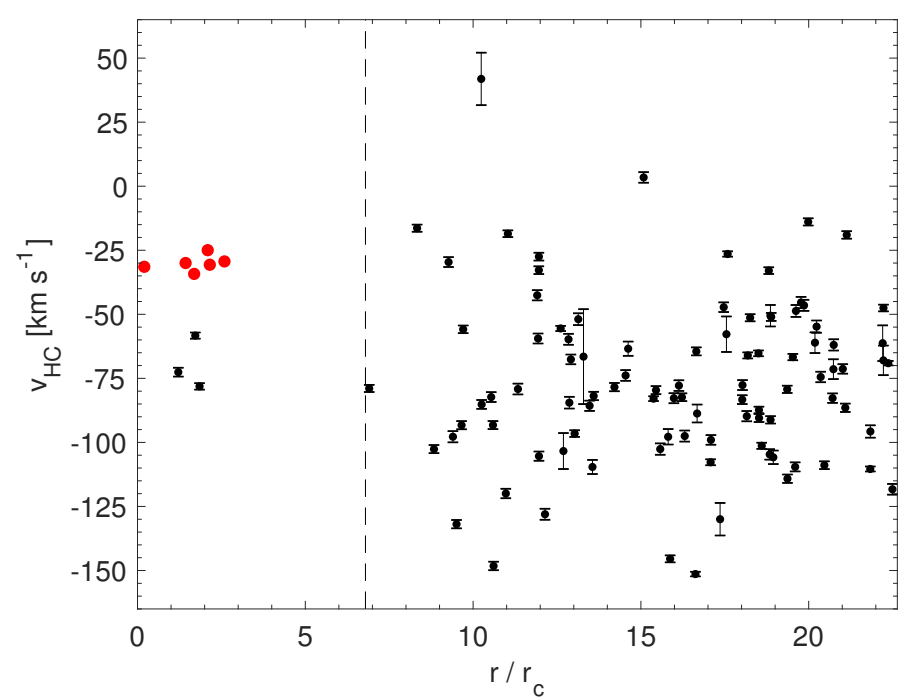

Fig. 2. Radial distribution of radial velocities in the inner sample, in units of the cluster's core-radius of 26.5" (Froebrich et al. 2008). The vertical line illustrates the "full extent" of the cluster of 3 ' as measured by Minniti et al. (2017). Red circles indicate the member candidates.

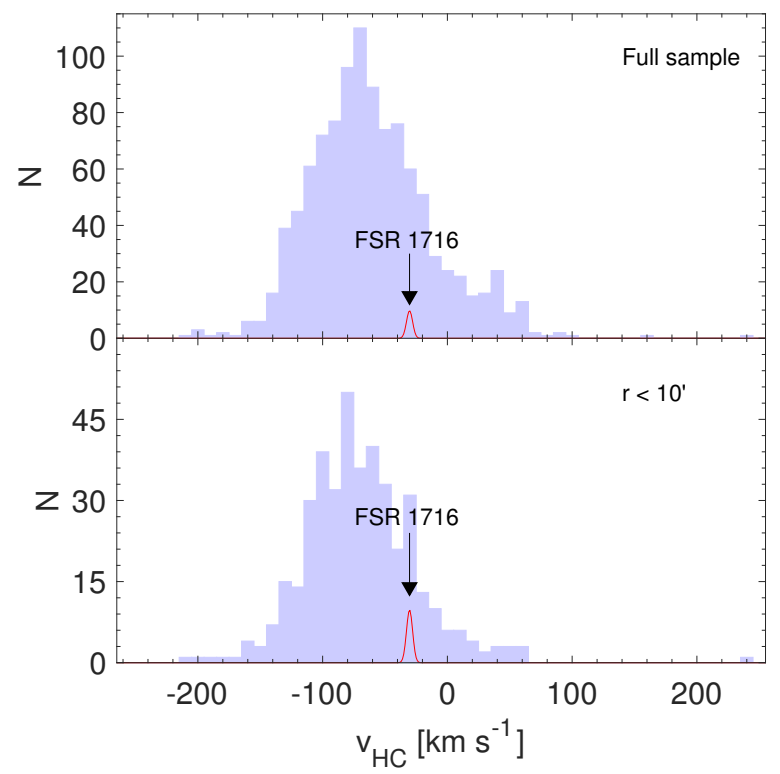

Fig. 3. Top panel: velocity histogram of the entire foreground sample including the six cluster member stars. Bottom panel: only stars with the inner $10^{\prime}$ to reflect the range shown in Fig. 2. The location of FSR 1716's mean velocity is indicated by an arrow.

via its core radius. For clarity, this has been truncated at $10^{\prime}$ to emphasize the "innermost" region of our data set close to the actual cluster.

As this figure and the distribution of the entire sample (Fig. 3) show, isolating the signal from the cluster itself is severely hampered by the Galactic foreground contamination. The mean velocities of these Galactic stars of $-61 \pm 2 \mathrm{~km} \mathrm{~s}^{-1}$ (heliocentric) and $-71 \pm 2 \mathrm{~km} \mathrm{~s}^{-1}$ (galactocentric) with a dispersion of $50 \pm 1 \mathrm{~km} \mathrm{~s}^{-1}$ are fully compatible with the Galactic rotation curve toward this line of sight (e.g., Dame et al. 2001).

We note, however, a group of six stars located within 2.6 core-radii of the center (red circles in Fig. 2) that show very similar kinematic properties, clustering around a mean velocity of $-30 \mathrm{~km} \mathrm{~s}^{-1}$. Likewise, as we will show in Sect. 4 , their the metallicities are in excellent agreement with each other. While
Table 2. Derived properties of the FSR 1716 member candidates.

\begin{tabular}{ccccc}
\hline \hline Star & $\begin{array}{c}S / N \\
{\left[\mathrm{px}^{-1}\right]}\end{array}$ & $\begin{array}{c}v_{\mathrm{HC}} \\
{\left[\mathrm{km} \mathrm{s}^{-1}\right]}\end{array}$ & $\begin{array}{c}\Sigma W \\
{[\AA]}\end{array}$ & $\begin{array}{c}{[\mathrm{Fe} / \mathrm{H}]_{\mathrm{CaT}}} \\
{[\mathrm{dex}]}\end{array}$ \\
\hline Plate 1 $\# 16$ & 26 & $-31.8 \pm 0.9$ & $4.40 \pm 0.11$ & $-1.38 \pm 0.20$ \\
Plate 1 \#24 & 40 & $-31.0 \pm 1.6$ & $4.92 \pm 0.08$ & $-1.28 \pm 0.20$ \\
Plate 1 \#106 & 20 & $-30.3 \pm 2.2$ & $4.19 \pm 0.15$ & $-1.37 \pm 0.21$ \\
Plate 2 \#266 & 34 & $-25.3 \pm 1.2$ & $3.98 \pm 0.17$ & $-1.51 \pm 0.21$ \\
Plate 3 \#76 & 28 & $-29.7 \pm 1.3$ & $4.98 \pm 0.11$ & $-1.23 \pm 0.20$ \\
Plate 3 \#213 & 18 & $-34.6 \pm 1.8$ & $3.46 \pm 0.17$ & $-1.64 \pm 0.21$ \\
\hline
\end{tabular}

the presence of a secondary plume of stars at $\sim-70 \mathrm{~km} \mathrm{~s}^{-1}$ could in principle be identified with an underlying cluster population as well, these stars have markedly different metallicities (Sect. 4) and we rather deem these foreground objects. The same holds for those stars outside of the cluster boundary of $\gtrsim 3^{\prime}$, Thus we identify the innermost six stars at the higher velocity peak with members of FSR 1716. Their individual properties are listed in Table 2.

Thereby, we establish the mean systemic velocity of FSR 1716 as $-30.3 \pm 1.2 \mathrm{~km} \mathrm{~s}^{-1}$ with a velocity dispersion of $2.5 \pm 0.9 \mathrm{~km} \mathrm{~s}^{-1}$, a moderately low value typical of a low-tointermediate mass Galactic star cluster (e.g., Pryor \& Meylan 1993; Koch et al. 2012, 2017b). Using this information and the cluster morphology in terms of its characteristic King (1966) radius (Froebrich et al. 2008; Bonatto \& Bica 2008; Minniti et al. 2017), we can estimate the dynamical mass of FSR 1716 as $\left(1.4_{-0.8}^{+1.2}\right) \times 10^{4} M_{\odot}$ (Spitzer 1987), which is a factor of $\sim 70$ larger than the low-mass estimate of Bonatto \& Bica (2008), albeit based on their premise of a significantly lower distance. Upon a typical stellar mass-to-light ratio of 1.5 , this would place FSR 1716 at an approximate absolute magnitude of $M_{V} \sim$ $-5.1 \pm 1.0 \mathrm{mag}$, without doubt in the regimen of a GC.

Ideally, the kinematic information should be coupled with accurate proper motions, allowing for an orbital computation that could hint at this cluster's origin. Unfortunately, none of the member stars had a cross-match in the UCAC5 catalog (Zacharias et al. 2017), and only three stars could be identified in the Hot Stuff for One Year (HSOY) proper motion catalog (Altmann et al. 2017), which used the most recent positions from Gaia-DR1 (Prusti et al. 2016). However, the respective proper motions are highly inconclusive, leading to a broad variety of possible orbits, from thick disk to outer halo; therefore, we do not pursue the orbital analyses any further.

\section{Calcium triplet metallicities}

The three near-infrared CaT lines are a powerful indicator of stellar metallicities, $[\mathrm{Fe} / \mathrm{H}]_{\mathrm{CaT}}$, for RGB stars near and above the horizontal branch (Armandroff \& Zinn 1988). In the following, we adopted the calibrations of line strength with metallicity from Vásquez et al. (2015), which are applicable into the metal-rich regime up to $0.7 \mathrm{dex}$, and which employ the infrared magnitude above the red clump $\left(K_{\mathrm{S}}-K_{\mathrm{RC}}\right)$. The latter is highly advantageous in population studies towards low latitudes, compared to the traditional use of the visual magnitude above the horizontal branch $\left(V-V_{\mathrm{HB}}\right)$, which is more sensitive to reddening. In practice, these calibrations are parameterized as

$[\mathrm{Fe} / \mathrm{H}]_{\mathrm{CaT}}=-3.15+0.432 W^{\prime}+0.006 W^{\prime 2}$,

with the reduced width $W^{\prime}=\Sigma E W+0.384\left(K_{\mathrm{S}}-K_{\mathrm{RC}}\right)$. Here, we defined the CaT line strength as the straight sum over the 


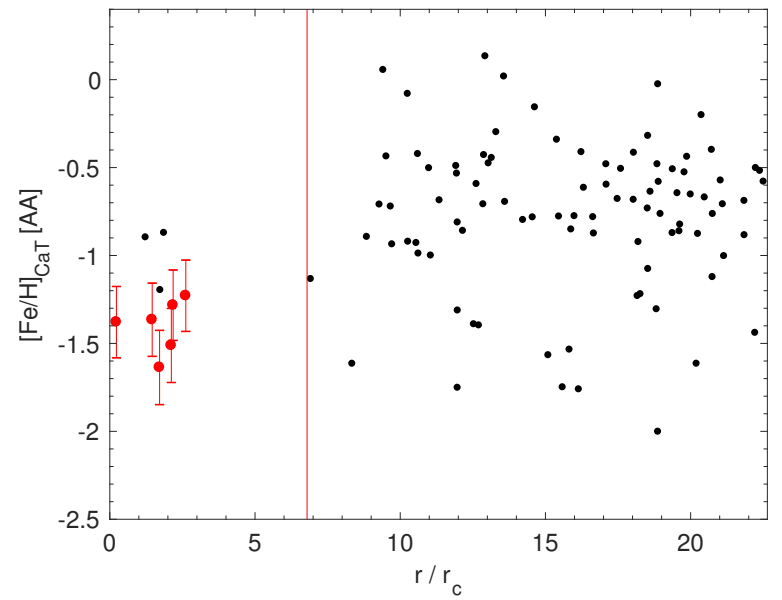

Fig. 4. CaT metallicities of the central parts of our observations. Member candidates are highlighted in red.

equivalent widths (EWs) of the two strongest lines, that is, $\Sigma E W=E W_{8542}+E W_{8662}$. The above formalism places our measurements on the metallicity scale of Carretta et al. (2009). The uncertainties on EW measurements and photometry were propagated through this process and added in quadrature to the rms scatter of 0.19 dex in the calibrations, as evaluated by Vásquez et al. (2015). The latter component also overwhelmed the overall error budget compared to the photometric and EW uncertainties.

We note that this procedure is explicitly only valid for stars above the red clump that are members to the system in question. Nominal metallicities assigned to foreground objects have no meaning, since their distance is a priori unknown so that the formal magnitude above the clump, introduced as a proxy for absolute magnitude, cannot be established. Furthermore, the $\mathrm{CaT}$ remains poorly measurable in many cold foreground stars in the sample due to the lines lying within strong molecular bands (Sharples et al. 1990; Kunder et al. 2012).

As Fig. 4, again truncated towards the inner $10^{\prime}$, shows, the velocity-selected member candidates share very similar CaTmetallicities, bolstering their identification with the cluster population. These stars are characterized by a mean metallicity of $-1.38 \pm 0.20 \mathrm{dex}$ and a $1 \sigma$ dispersion of $0.15 \mathrm{dex}$ that is purely driven by the measurement errors that are typically of 0.20 dex. This is in excellent agreement with previous measurements of the cluster mean from infrared photometry $\left(-1.6_{-0.6}^{+0.3}\right.$; Froebrich et al. 2008). Likewise, Minniti et al. (2017) performed CMD-fitting on their VVV infrared photometry and used the period-metallicity relation for RR-Lyrae to ascertain a value of $-1.5 \pm 0.4$ dex. Combined with its kinematic properties and the appearance of its CMD, this argues in favor of this system indeed being an old, metal-poor GC, ruling out a younger open disk cluster or a Solar-metallicity object as suggested by Froebrich et al. (2008) and Bonatto \& Bica (2008).

\section{Conclusions}

The low-latitude star cluster FSR 1716 has so far defied a clearcut identification and has been placed in various classes since its discovery. While originally suspected as an old open cluster at a close distance and very low mass of only a few hundreds solar masses (Froebrich et al. 2007; Bonatto \& Bica 2008), the recent detection of old RR Lyrae stars associated with this object has argued in favor of a location near the Galactic bulge and an even older age, suggesting a globular nature.

The present spectroscopic study has firmly established FSR 1716 as a metal-poor system (at -1.4 dex), in line with photometric measurements. Our kinematic measurements confirmed a low mass on the order of $10^{4} M_{\odot}$, thus rendering this object an old, metal-poor and low-mass globular cluster that is currently located in the inner disk of the Milky Way. Clearly, improved proper motions that can be gleaned from long-baseline, high-accuracy surveys (e.g., Zacharias et al. 2017; Minniti et al. 2017) are needed to aid in further investigation the history of this intriguing object.

Acknowledgements. We are grateful to the referee, Jeffrey Simpson, for a very swift and helpful report. Based on data acquired through the Australian Astronomical Observatory, via program NOAO/0195 (PI: A. Kunder). The research leading to these results has received funding from the European Community's Seventh Framework Programme (FP7/2013-2016) under grant agreement number 312430 (OPTICON, proposal 17A/051). This work was supported in parts by Sonderforschungsbereich SFB 881 "The Milky Way System" (subproject A8) of the German Research Foundation (DFG).

\section{References}

AAO software Team 2015, 2dfdr: Data reduction software, Astrophysics Source Code Library [record ascl : 1505.015]

Altmann, M., Roeser, S., Demleitner, M., Bastian, U., \& Schilbach, E. 2017, A\&A, 600, L4

Armandroff, T. E., \& Zinn, R. 1988, AJ, 96, 92

Bonatto, C., \& Bica, E. 2007, A\&A, 473, 445

Bonatto, C., \& Bica, E. 2008, A\&A, 491, 767

Brunthaler, A., Reid, M. J., Menten, K. M., et al. 2011, Astron. Nachr., 332, 461

Buckner, A. S. M., \& Froebrich, D. 2016, ArXiv e-prints [arXiv: 1611.03753]

Carretta, E., Bragaglia, A., Gratton, R., D’Orazi, V., \& Lucatello, S. 2009, A\&A, 508,695

Cutri, R. M., Skrutskie, M. F., van Dyk, S., et al. 2003, 2MASS All Sky Catalog of point sources. The IRSA 2 MASS, All-Sky Point Source Catalog, NASA/IPAC Infrared Science Archive

Dame, T. M., Hartmann, D., \& Thaddeus, P. 2001, ApJ, 547, 792

Dotter, A., Chaboyer, B., Jevremović, D., et al. 2008, ApJS, 178, 89

Friel, E. D. 2013, Open Clusters and Their Role in the Galaxy, eds. T. D. Oswalt, \& G. Gilmore, 347

Froebrich, D., Scholz, A., \& Raftery, C. L. 2007, MNRAS, 374, 399

Froebrich, D., Meusinger, H., \& Scholz, A. 2008, MNRAS, 390, 1598

Ivanov, V. D., Kurtev, R., \& Borissova, J. 2005, A\&A, 442, 195

King, I. R. 1966, AJ, 71, 64

Kleyna, J. T., Wilkinson, M. I., Evans, N. W., \& Gilmore, G. 2004, MNRAS, 354, L66

Koch, A., Lépine, S., \& Çalışkan, Ş. 2012, in EPJ Web Conf., 19, 03002

Koch, A., McWilliam, A., Preston, G. W., \& Thompson, I. B. 2016, A\&A, 587, A124

Koch, A., Hansen, C. J., \& Kunder, A. 2017a, A\&A, 604, A41

Koch, A., Hansen, T. T., \& Kunder, A. 2017b, A\&A, submitted [arXiv: 1709. 04022]

Kunder, A., Koch, A., Rich, R. M., et al. 2012, AJ, 143, 57

Lamers, H. J. G. L. M., Gieles, M., Bastian, N., et al. 2005, A\&A, 441, 117

Law, D. R., \& Majewski, S. R. 2010, ApJ, 718, 1128

Marín-Franch, A., Aparicio, A., Piotto, G., et al. 2009, ApJ, 694, 1498

Minniti, D., Palma, T., Dékány, I., et al. 2017, ApJ, 838, L14

Mucciarelli, A., Monaco, L., Bonifacio, P., \& Saviane, I. 2017, A\&A, 603, L7

Prusti, T., de Bruijne, J. H. J., Brown, A. G. A., et al. 2016, A\&A, 595, A1

Pryor, C., \& Meylan, G. 1993, in Structure and Dynamics of Globular Clusters, eds. S. G. Djorgovski, \& G. Meylan, ASP Conf. Ser., 50, 357

Recio-Blanco, A., de Laverny, P., Kordopatis, G., et al. 2014, A\&A, 567, A5

Reid, M. J., Menten, K. M., Zheng, X. W., et al. 2009, ApJ, 700, 137

Sharp, R., Saunders, W., Smith, G., et al. 2006, in SPIE Conf. Ser., Proc. SPIE, 6269, 62690G

Sharples, R., Walker, A., \& Cropper, M. 1990, MNRAS, 246, 54

Spitzer, L. 1987, Dynamical evolution of globular clusters (Princeton, NJ: Princeton University Press)

Vande Putte, D., Garnier, T. P., Ferreras, I., Mignani, R. P., \& Cropper, M. 2010, MNRAS, 407, 2109

Vásquez, S., Zoccali, M., Hill, V., et al. 2015, A\&A, 580, A121

Zacharias, N., Finch, C., \& Frouard, J. 2017, AJ, 153, 166 